\title{
Generation of a recombinant rabies Flury LEP virus carrying an additional $G$ gene creates an improved seed virus for inactivated vaccine production
}

Lihong Tao ${ }^{1}$, Jinying Ge${ }^{1}$, Xijun Wang ${ }^{1}$, Zhiyuan Wen ${ }^{1}$, Hongyue Zhai ${ }^{1}$, Tao Hua', Bolin Zhao ${ }^{1}$, Dongni Kong ${ }^{1}$, Chinglai Yang ${ }^{2}$ and Zhigao $\mathrm{Bu}^{1 *}$

\begin{abstract}
The rabies Flury Low Egg Passage virus (LEP) has been widely used as a seed virus to generate inactive vaccine. Here, we established a reverse genetic system for LEP and generated a recombinant LEP virus (rLEP-G) that carries two identical $G$ genes. This recombinant virus showed similar properties to those of LEP with respect to in vitro growth, neurotropism index, and virulence in mice. rLEP-G produced 4.3-fold more G protein than did LEP in BHK21 cells. The inactivated vaccine generated from rLEP-G induced significantly higher virus neutralization titers in mice and dogs than those produced in response to LEP-derived vaccine. Our results suggest that rLEP-G is an improved seed virus candidate for inactivated rabies virus vaccine manufacture.
\end{abstract}

Keywords: Rabies virus, LEP, recombinant, inactivated vaccine

\section{Introduction}

Rabies virus (RV) belongs to the genus Lyssavirus of the family Rhabdoviridae, and causes a fatal neurological disease in humans and animals [1] More than 55,000 people die of rabies each year, and about $95 \%$ of these deaths occur in Asia and Africa [2]. An estimated 31,000 people die from dog rabies in Asia each year, with most cases occurring in India and China [3,4]. The most cost-effective strategy for preventing rabies in people is to eliminate rabies in dogs via vaccination [5-7] Inactivated rabies vaccine has been shown to be a safe and efficient means to control rabies in dogs. However, the vaccination rate of dogs in many developing countries is low, especially in rural areas, mainly due to low economic development and the high cost of vaccination [8] More efficient and lower cost inactivated vaccine is, therefore, still needed.

\footnotetext{
* Correspondence: zgbu@yahoo.com

${ }^{1}$ State Key Laboratory of Veterinary Biotechnology, Harbin Veterinary Research Institute of Chinese Academy of Agricultural Sciences, Harbin 150001, P.R. China

Full list of author information is available at the end of the article
}

The surface glycoprotein (G) of RV is the major antigen responsible for the induction of protective immunity [1] Increasing $G$ protein levels should, therefore, enhance the protective viral neutralization antibody (VNA) response. The rabies Flury low egg passage virus (LEP) has been widely used as a seed virus to generate inactive vaccine for humans and animals because of its high immunogenicity and high growth titer in cell culture [9]. Here, we generated a recombinant LEP virus that carries two identical $G$ genes to increase $G$ protein expression. Growth curves, neurotropism index, virulence, and the $\mathrm{G}$ protein expression level of the doubleG LEP were tested in vitro and in vivo. The immunogenicity of the inactivated vaccine derived from this double-G LEP was also evaluated in mice and dogs and compared with that of LEP.

\section{Materials and Methods Viruses and cells}

Neuroblastoma (NA) cells of A/J mouse origin were grown in Eagle's minimum essential medium (MEM) supplemented with $10 \%$ fetal bovine serum (FBS). Baby hamster kidney (BHK-21) cells were grown in 
Dulbecco's modified Eagle's MEM (DMEM) supplemented with 10\% FBS. The RV LEP (AV2012) was obtained from the China Veterinary Culture Collection Center and propagated in BHK-21 cells. A street virus, GX/09, was isolated from the brain of a dog that died of rabies and was propagated in the brain of adult mice. All viruses were kept in at $-70^{\circ} \mathrm{C}$ before use.

\section{Plasmids construction}

Viral RNA was extracted with an RNeasy mini kit according to the manufacturer's instructions (QIAGEN, Valencia, CA). The extracted RNA was subjected to RTPCR with virus specific primer pairs (Table 1) and highfidelity $P f x$ DNA polymerase (Invitrogen Corp., Carlsbad, CA) to generate three overlapping PCR fragments (F1, F2, and F3) that encompassed the entire viral genome. The assembled cDNA, containing the hammerhead ribozyme sequence (HamRz), the full-length $(11,925$ nucleotide) cDNA of the LEP strain genome in the antigenomic orientation, and the hepatitis delta virus ribozyme sequence ( $\mathrm{HdvRz})$, was inserted between the Nhe I and Sma I sites of pCI. A Pme I restriction site was introduced into the G-L noncoding region by changing three nucleotide residues at positions 4907 ( $\mathrm{T}$ to $\mathrm{G}$ ), 4910 ( $\mathrm{G}$ to $\mathrm{T}$ ) and 4912 ( $\mathrm{C}$ to $\mathrm{A}$ ) by using a site-directed mutagenesis system (Invitrogen) with the primers shown in Table 1 . The resultant plasmid was designated as pLEP. The cDNA of 1,801 nucleotides including the open reading frame of the $G$ gene was amplified from pLEP by the primer pair shown in Table 1 . The fragment was introduced into the LEP genome through the Pme I site. The resultant plasmid was designated as pLEP-G (Figure 1). The open reading frames (ORFs) of the N, P, and L genes were PCR-amplified from pLEP-G with the primers shown in Table 1 for the construction of the N, P, and L expression plasmids. The amplified $\mathrm{N}, \mathrm{P}$, and L genes were inserted between the EcoR I and Kpn I sites in the plasmid pCAGGS and were designated as pCA-N, pCA-P, and pCA-L, respectively. The assembled full-length cDNA clone and the helper plasmids were sequenced in their entirety to ensure that no undesirable mutations had been introduced.

\section{Virus rescue}

BHK-21 cells were grown overnight to $80 \%$ confluence in 6-well plates in DMEM supplemented with 10\% FBS. Cells were transfected with $4.0 \mu \mathrm{g}$ of the full-length plasmid pLEP-G, $2 \mu \mathrm{g}$ of pCA-N, $1 \mu \mathrm{g}$ of pCA-P, and 1 $\mu \mathrm{g}$ of pCA-L by using Lipofectamine ${ }^{\mathrm{TM}} 2000$ (Invitrogen) according to the manufacturer's protocol. After 4-6 $h$, the transfection medium was replaced with fresh medium. After 3 days, supernatants were transferred onto BHK-21cells and incubated for a further 3 days. Rescued viruses were examined by using an indirect fluorescence assay (IFA) with mouse anti-RV serum and FITC-conjugated goat anti-mouse IgG (Sigma). Supernatants from virus-positive cultures were collected to propagate virus stocks in BHK-21 cells. Sequences of recovered viruses were confirmed by sequencing entire viral genomes. To confirm whether the recovered rabies virus was derived from the cloned PLEP-G, RT-PCR was performed with the primers shown in Table 1, positivesense G4 and negative-sense G3 primers at nucleotide positions 4,302 to 4,321 and 3,613 to 3,632 (based on the genomic nucleotide number of the LEP strain), respectively. The fragment was further digested with Pme I, and the supernatants from virus-positive cultures were used to produce virus stocks in BHK-21 cells. The rescued virus generated from the full-length plasmid pLEP-G was designated as rLEP-G.

\section{Multistep growth assays}

Confluent BHK-21 or NA cell monolayers grown in 6well plates were infected with LEP or rLEP-G at a multiplicity of infection (MOI) of 0.01. After incubation for 1 $h$ at $37^{\circ} \mathrm{C}$, the inoculums were removed and the cells were washed twice with PBS. BHK-21 and NA cells were replenished with DMEM containing 2\% FBS or with MEM containing $0.2 \%$ FBS, respectively, and incubated at $37^{\circ} \mathrm{C}$. Culture supernatant $(100 \mu \mathrm{l})$ was harvested at $0,1,2,3,4$, and 5 days post-inoculation for virus titration.

\section{Virus titration}

Monolayers of NA or BHK-21 cells in 24-well plates were infected with 10 -fold dilutions of virus suspension and incubated at $37^{\circ} \mathrm{C}$. At $48 \mathrm{~h}$ post-infection, an IFA test was performed. Foci were counted under a fluorescence microscope and calculated as focus forming unit/ $\mathrm{ml}(\mathrm{FFU} / \mathrm{ml})$.

The in vitro neurotropism index was expressed as the logarithm of the titer of virus stock in NA cells minus the logarithm of the titer of the same stock in BHK-21 cells [10]. The virus titers for neurotropism index calculation were based on the titration results of virus stock harvested at $72 \mathrm{~h}$ post infection.

\section{Western blotting}

BHK-21 cells grown in 6-well plates were infected with LEP or rLEP-G at an MOI of 1 and incubated for $48 \mathrm{~h}$. $\mathrm{G}$ gene expression was confirmed by using western blotting as described previously [11]. Briefly, cell extracts were subjected to $10 \%$ polyacrylamide gel electrophoresis (SDS-PAGE), and then blotted to nitrocellulose membrane. The membrane was incubated with a mixture of mouse anti-G protein polyclonal antiserum and anti- $\beta$-actin monoclonal antibody (Santa Cruz Biotechnology), as the first antibody, and then with IRDye700- 
Table 1 Primers used to construct the full-length CDNA clone and helper plasmids for Flury LEP

\begin{tabular}{|c|c|c|}
\hline Purpose & Name & Primer $\left(5^{\prime}-3^{\prime}\right)^{\mathrm{a}}$ \\
\hline \multirow[t]{3}{*}{ For F1 } & amplification & F1-F \\
\hline & & TGCGCTAGCTGTTAAGCGTCTGATGAGTCCGTGAGGACGAAACTATAGGAAAGGAATTCCTATAGTCACGCTTAACAACAAAACCAAAGAA ${ }^{\mathrm{b}}$ \\
\hline & F1-R & GGCACGCGTACTCCACATAACTTGAGTTTGC \\
\hline \multirow[t]{3}{*}{ For F2 } & amplification & F2-F \\
\hline & & AGGCCTGTATAAGTCTTTAAAGGGAGCA \\
\hline & $\mathrm{F} 2-\mathrm{R}$ & ATCGGGGTTCCCGGCCTCTTGACACAAC \\
\hline \multirow[t]{3}{*}{ For F3 } & amplification & F3-F \\
\hline & & TATGCTAGCTCTGGTTAAGCTCCCACGAATC \\
\hline & F3-R & CGATCCCGGGccccgcgggggeccctcccttagccatccgagtggacgaacgtcctccttcggatgcccaggtcggaccgcgaggaggtggagatgccatgccgacccACGCTTAACAAATAAACAAT \\
\hline \multirow{2}{*}{$\begin{array}{l}\text { For Pme I } \\
\text { mutation }\end{array}$} & Pmu-F & GACTTGAAGTITAAACAGGATGACCGGCC \\
\hline & Pmu-R & GGCCGGTCATCCTGTITIAAACTTCAAGTC \\
\hline \multirow{3}{*}{$\begin{array}{l}\text { For G } \\
\text { gene }\end{array}$} & amplification & G1 \\
\hline & & ATGCGTTTAAACAAGTTTATCACTTGTTTACCTCT \\
\hline & G2 & GCATGTTTAAACACTTGAAGTGTCAAAAGGATGA \\
\hline \multirow{3}{*}{$\begin{array}{l}\text { For } N \\
\text { gene }\end{array}$} & amplification & $\mathrm{HN}-\mathrm{F}$ \\
\hline & & GGCGAATTCATGGATGCCGACAAGATTGT \\
\hline & HN-R & CCGGGTACCTTATGAGTCACTCGAATACG \\
\hline \multirow[t]{3}{*}{ For L gene } & amplification & $\mathrm{HL}-\mathrm{F}$ \\
\hline & & GGCGAATTCATGCTGGATCCGGGAGAGGTT \\
\hline & $H L-R$ & CCGGGTACCTTACAAACAACTGTAGTCTA \\
\hline \multirow[t]{3}{*}{ For LEP-G } & confirmation & G3 \\
\hline & & ATGCTITCTCTTGAATGTGG \\
\hline & G4 & GGGTTTGGAAAAGCATATAC \\
\hline
\end{tabular}




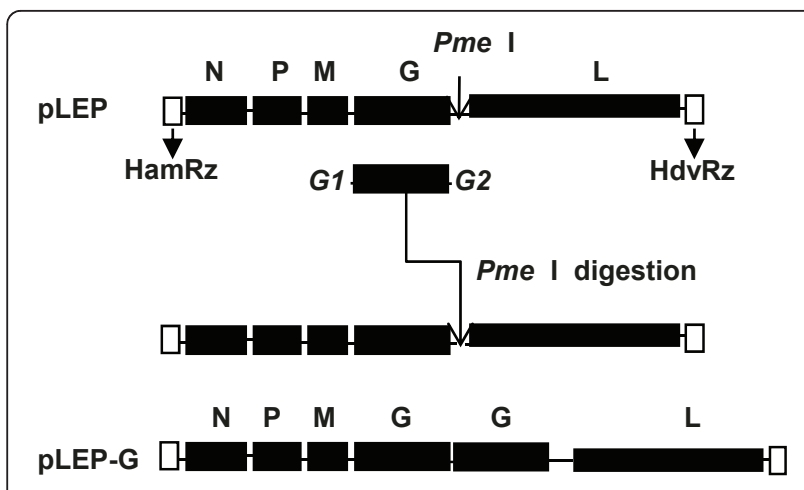

Figure 1 Schematic representation of recombinant virus containing two homologous $\mathrm{G}$ genes. The $\mathrm{PLEP}$ vector was obtained by assembling three overlapping CDNA fragments of the Flury LEP strain into the vector $\mathrm{pCl}$, as described in the Methods. The 1801-bp fragment including the G gene ORF was introduced into the LEP genome through the Pme I site in the G-L noncoding region. The hammerhead ribozyme sequence (HamRz) was introduced upstream of the LEP genome, and the hepatitis delta virus ribozyme sequence ( $\mathrm{HdvRz}$ ) was introduced downstream of the viral genome.

conjugated anti-mouse IgG (Rockland, Gilbertsville, PA) as the second antibody. The mouse anti-G protein polyclonal antiserum was generated by immunization with a recombinant Newcastle disease virus that expresses the RV glycoprotein and was developed in our lab. Signal intensities were analyzed by using the Odyssey infrared image system (LiCor). The densitometry of each band was quantified with Photoshop CS2. The G/ $\beta$-actin ratio was calculated by dividing the densitometry of the $G$ protein band by that of the $\beta$-actin protein band.

\section{Infection of mice}

The virulence of LEP and rLEP-G for adult mice was measured in 6-week-old female Balb/c mice (Vital River, Beijing). To determine the $50 \%$ lethal dose $\left(\mathrm{LD}_{50}\right)$ of each virus, groups of five adult mice were inoculated intracerebrally (i.c.) with $30 \mu \mathrm{l}$ or intramuscularly (i.m.) with $100 \mu \mathrm{l}$ of serial 10-fold dilutions of LEP or rLEP-G. After infection, mice were observed for 21 days for clinical signs or death. The $\mathrm{LD}_{50}$ of each virus was calculated by using the method of Reed and Muench [12].

\section{Preparation of inactivated vaccines}

BHK-21 cells grown in $75 \mathrm{~cm}^{2}$ flask were infected with LEP or rLEP-G at an MOI of 0.01 and incubated at $37^{\circ}$ C. At $96 \mathrm{~h}$ post-infection, cells were suspended in the supernatant by using a rubber policeman. The suspension was submitted to three cycles of freezing and thawing $\left(-20^{\circ} \mathrm{C} / 37^{\circ} \mathrm{C}, 5 \mathrm{~min}\right.$ each) and then subjected to ultrasonic sound to increase the cell extraction dissolution. After centrifuge, supernatants were collected and stored in $-70^{\circ} \mathrm{C}$. The infectious viruses in the supernatant were titrated in BHK cells. To prepare inactivated vaccines, the supernatant was incubated at $37^{\circ} \mathrm{C}$ for $2 \mathrm{~h}$ in the presence of $0.03 \% \beta$-propiolactone. Then, three volumes of inactivated supernatant preparation (non-dilution, 20-f old dilution, and 80-fold dilution) were mixed with one volume of Rehydraphos $\mathrm{AlPO}_{4}$ adjuvant (Reheis, Berkeley Heights, NJ), and stored at $4^{\circ}$ $\mathrm{C}$ for vaccination.

\section{Immunization of Balb/c mice and dogs}

Groups of ten 4-week-old female Balb/c mice were inoculated i.m. in the gastrocnemius muscle with $100 \mu \mathrm{l}$ of 20- or 80 -fold dilutions of inactivated vaccine preparations. Groups of six 3-month-old Beagles were inoculated $i . m$. with $1 \mathrm{ml}$ of inactivated vaccine preparations.

\section{VNA assay}

Three weeks after immunization, mice were bled from the retro-orbital sinus under isoflurane inhalation anesthesia and Beagles were bled from the vein of the front leg. Sera were tested for neutralizing antibodies (VNA) by using the rapid fluorescent focus inhibition test (RFFIT) [13]. Neutralization titers, defined as the highest serum dilution that neutralizes $50 \%$ of the challenge virus, were normalized to international units (IU) according to the World Health Organization anti-RV antibody standard.

\section{Results}

Recovery of rLEP-G from cloned CDNA

The full-length genome plasmids of recombinant LEP carrying two identical $G$ genes were assembled and used to rescue recombinant virus. The resulting virus was designated as rLEP-G. To exclude the possibility of contamination with LEP, a cDNA fragment covering the G$G$ noncoding region was amplified from the genomic RNA of the rescued recombinant virus and LEP, respectively, by using RT-PCR with a set of primers, G3 and G4. The amplified cDNA fragments were digested with Pme I. A fragment with the expected size of $1,125 \mathrm{bp}$ was amplified from the genomic RNA of rLEP-G, whereas RT-PCR with the genomic RNA of LEP did not amplify any detectable product (Figure 2A). The amplified cDNA fragment was digested with Pme I, and 610bp and 515-bp bands were observed (Figure 2B). Sequence analysis further confirmed that the virus was rescued from cloned pLEP-G. RV G protein expression by rLEP-G in BHK-21 cells was compared with that of LEP by using Western blot analysis. G protein was expressed at a significantly higher level in rLEP-Ginfected cells than in LEP-infected cells (Figure 2C). In LEP-infected cells, the $G / \beta$-actin ratio was 1.2 , whereas in rLEP-G-infected cells the $\mathrm{G} / \beta$-actin ratio was 5.1 , 


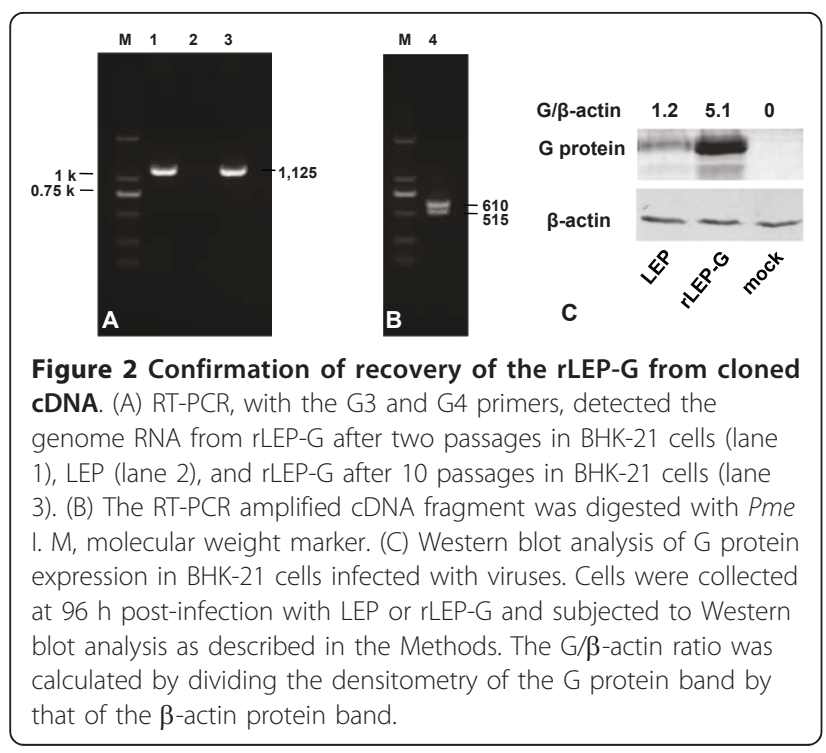

indicating an approximate 4.3-fold increase in G protein levels in rLEP-G-infected cells due to expression from the additional $\mathrm{G}$ gene.

\section{Growth properties of rLEP-G in vitro}

To examine whether insertion an identical $G$ gene into the genome of the LEP strain would affect the growth properties of the virus in vitro, we compared the growth of rLEP-G and LEP in both neuronal NA cells and nonneuronal BHK-21 cells. The multistep growth curves of rLEP-G (the third passage generation from BHK-21 cells) were compared with that of LEP in NA cells. There were no significant differences in growth patterns in both cell lines (Figure 3A, B) between rLEP-G and LEP. Furthermore, LEP and rLEP-G showed similar in vitro neurotropism indices of 0.84 and 0.85 , respectively, which indicates that the additional G gene did not affect the in vitro neurotropism of the LEP strain.

To examine the genome stability of rLEP-G in cell culture, the virus was serially passaged in BHK-21 cells 10 times. RT-PCR analysis revealed that the additional
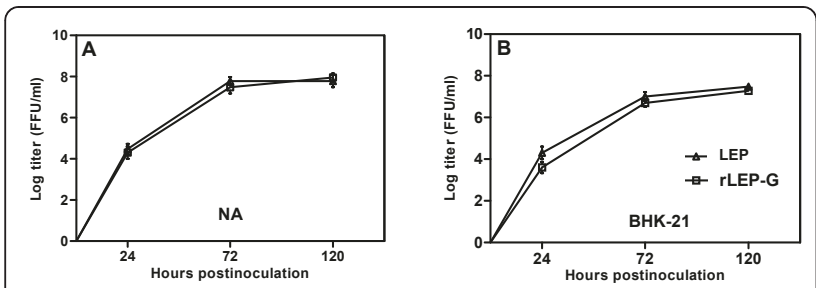

Figure 3 Multistep growth curves for LEP and rLEP-G. NA cells (A) and BHK-21 Cells (B) were infected at an $\mathrm{MOI}$ of 0.01 . The infected cell culture supernatants were harvested at different time post-inoculation, and virus titers were determined in triplicate in BHK-21 cells.
G gene in the rLEP-G genome was stable for at least 10 passages in BHK-21 cells. The titer of rLEP-G also remained high at approximately $1 \times 10^{8} \mathrm{FFU} / \mathrm{ml}$, from the third to the tenth passage (Figure 4).

\section{Pathogenicity of rLEP-G in adult mice}

The $G$ protein plays an important role in the pathogenicity of RV. Therefore, we needed to investigate whether insertion of an additional $\mathrm{G}$ gene would affect virulence. Accordingly, adult mice were inoculated with rLEP-G and LEP, respectively. The results showed that both rLEP-G and LEP caused neurological symptoms, such as paralysis and hyperactivity, and killed mice after i.c. inoculation. The $\mathrm{LD}_{50}$ of rLEP-G following i.c. inoculation was $2 \mathrm{FFU}$, which was 2-fold higher than that of LEP (Figure 5). Peripheral pathogenicity was also examined following i.m. inoculation. All mice survived i.m. inoculation with $10^{6} \mathrm{FFU}$ of rLEP-G, whereas, one of the five mice inoculated i.m. with the same dose of LEP died. This result indicates that the in vivo neuro-invasiveness and peripheral pathogenicity of LEP did not increase after insertion of the additional G gene.

\section{Induction of VNA in mice and dogs}

VNA is mainly induced by viral antigen $G$ protein and plays a key role in immune protection from rabies. To investigate whether increased expression of $G$ in the seed virus improves the VNA response of inactivated rabies vaccine, two inactivated vaccines were respectively preparations from LEP and rLEP-G infected cell cultures as described in materials and methods. The virus titers in supernatant of LEP and rLEP-G infected cell cultures were $10^{7.4} \mathrm{FFU} / \mathrm{ml}$ and $10^{7.3} \mathrm{FFU} / \mathrm{ml}$ respectively. The preparations were then tested in adult mice by using i.m.

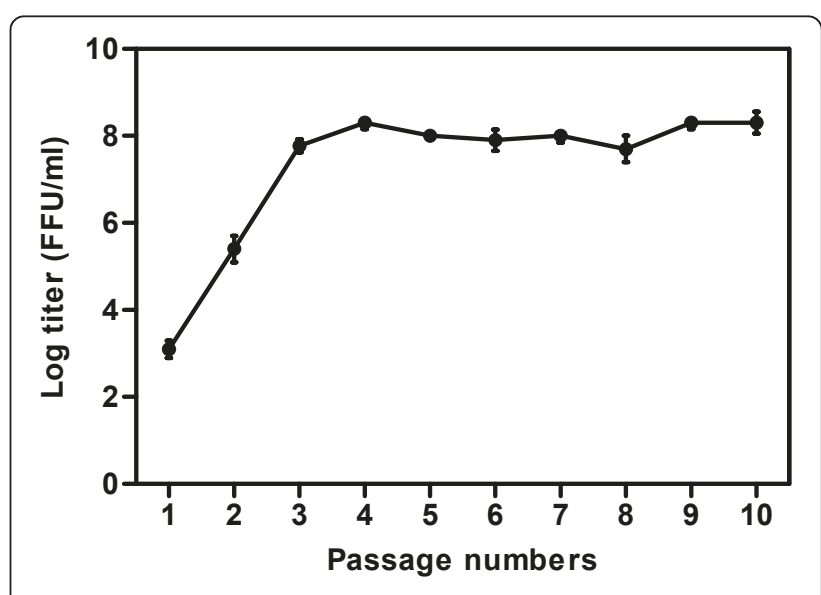

Figure 4 Virus titers of rLEP-G serially propagated in BHK-21 cells. BHK-21 cells were infected with rLEP-G at an $\mathrm{MOI}$ of 0.01 . The infected cell culture supernatants from each passage were harvested at $96 \mathrm{~h}$ post-inoculation, and virus titers were determined in triplicate in BHK-21 cells. 

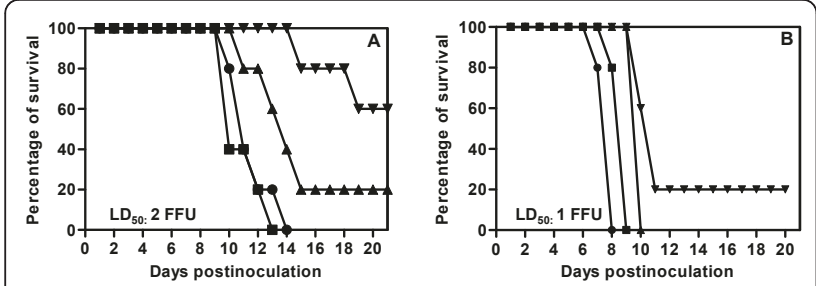

Figure 5 Pathogenicity of LEP and rLEP-G in adult mice. Sixweek-old Balb/c mice were inoculated intracerebrally with LEP (A) or rLEP-G (B) in a $30 \mu \mathrm{l}$ volume at doses of $10^{0}$ to $10^{3} \mathrm{FFU}$ (black inverted triangle: $10^{0} \mathrm{FFU}$; black triangle: $10^{1} \mathrm{FFU}$; black square: $10^{2}$ FFU; black dot: $10^{3} \mathrm{FFU}$ ). After infection, mice were observed for 21 days for clinical signs or death. The MLD 50 of each virus was calculated by using the method of Reed and Muench.

inoculation with. There were no significant differences in VNA response between the mice that received $0.1 \mathrm{ml}$ of 20 -fold diluted LEP vaccine preparation (containing 5.2 $\log _{10} \mathrm{FFU} / 0.1 \mathrm{ml}$ ) and those that received $0.1 \mathrm{ml}$ of 20 fold diluted LEP-G vaccine preparation (for LEP and 5.1 $\log _{10}$ FFU/0.1 ml for rLEP-G). However, the VNA of mice immunized with an 80-fold diluted rLEP-G vaccine preparation (containing $4.5 \log _{10} \mathrm{FFU} / 0.1 \mathrm{ml}$ ) was significantly higher than that of mice immunized with a similarly diluted LEP vaccine preparation (containing 4.4 $\log _{10}$ FFU/0.1 ml) $(\mathrm{P}<0.01)$ (Figure 6A). In dogs, $1 \mathrm{ml}$ of rLEP-G vaccine preparation (containing $7.3 \log _{10} \mathrm{FFU} / \mathrm{ml}$ ) induced a VNA mean titer of 54 IU, which was significantly higher than mean titer of 15 IU that induced by 1 $\mathrm{ml}$ of LEP inactivated vaccine (containing $7.2 \log _{10} \mathrm{FFU} /$ $\mathrm{ml})(\mathrm{P}<0.05)$ (Figure 6B). These results indicate that the rLEP-G strain is a more immunogenic seed virus for use in the development of inactive vaccines against rabies.

\section{Discussion}

Mass vaccination of dogs has proven to be an effective way to control rabies in this species. However, current
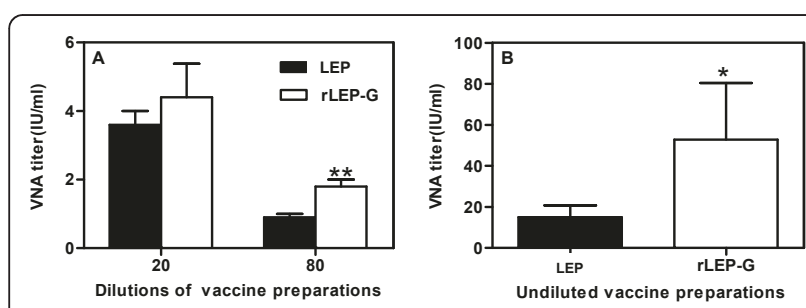

Figure 6 VNA titers in mice and dogs immunized with inactivated LEP or rLEP-G vaccine preparations. (A) Groups of 10 mice were immunized with 20- or 80-fold dilutions of inactivated vaccine preparations of LEP or rLEP-G. (B) Groups of 6 dogs were immunized with $1 \mathrm{ml}$ of inactivated vaccine preparations of LEP or rLEP-G. Blood samples were collected at 3 weeks post-vaccination and subjected to the VNA titration test. VNA were determined by using the rapid fluorescence inhibition test. Titers were normalized to international units by using the World Health Organization standard. ${ }^{* *}, P<0.01 ;{ }^{*}, P<0.05$. live attenuated vaccines can have safety issues $[14,15]$, and the use of current inactivated vaccines is limited in developing countries because of high manufacturing costs. Increasing $\mathrm{G}$ protein expression in RV seed viruses is hypothesized to improve rabies inactivated vaccines not only via immunogenicity but also through increased manufacturing productivity. Previous studies have demonstrated the feasibility of introducing an additional G gene into the RV genome [16-18]. By using reverse genetics technology, Faber et al. generated a double-G gene recombinant RV (SPBNGA-GA). This double-G virus showed a 1.6-fold increase in $G$ protein expression in cell culture relative to that of its single-G gene parent virus (SPBNGA). Inoculation with SPBNGA-GA live virus provided more efficient protective immunity than did the SPBNGA strain in mice. However, the utility of SPBNGA-GA as a seed virus for inactivated vaccine production has not been evaluated further [18]. In another study, Hosokawa-Muto et al. generated a double-G gene recombinant RV RC-HL. This virus showed a 1.5 -fold increase in $G$ protein expression in cell culture compared with that induced in the single-G gene parent virus RC-HL[16]. When the recombinant double-G gene RC-HL and wild-type RCHL were inactivated and used to vaccinated mice, no significant difference in RV neutralization antibody responses was observed.

Here, we generated a recombinant LEP virus carrying an additional G gene, rLEP-G. This recombinant virus produced strikingly higher levels of $\mathrm{G}$ protein in cell culture and showed similar in vitro growth properties and bio-safety characteristics. Inactivated vaccine prepared from rLEP-G induced significant higher RV VNA titers in mice and dogs than those induced by LEPderived vaccine, indicating that $\mathrm{RLEP}-\mathrm{G}$ represents an improved seed virus candidate for inactivated RV vaccine manufacture.

Since G protein is a major contributor to the pathogenicity of rabies virus [19-22], we evaluated whether this increase in $G$ protein expression affected the virulence of the rLEP-G virus. We found that the insertion of this additional $G$ gene results in increase of $G$ protein expression but not in increase of the neuro-invasiveness or peripheral pathogenicity of the virus in adult mice. In other studies, introduction of one or two additional G genes into the genome of rabies virus resulted in a higher level of $G$ protein expression and attenuate pathogenicity in adult mice $[17,18,23]$. As the pathogenicity of a particular RV strain correlates inversely with its ability to trigger apoptosis in neuron cells[23], one possible explanation for this discrepancy is that some lethal viruses, such as LEP and rLEP-G, rarely induced apoptosis in neuron cells. In fact, our previous data had shown that there was no clear correlation between apoptosis 
induction and the viral replication titers or glycoprotein expression level among LEP and its relative mutants or chimerical viruses[19].

\section{Acknowledgements}

We thank Susan Watson for editing the manuscript. This work was supported by Chinese National S\&T Plan (2009ZX10004-214), by the grant from Chinese Ministry of Agriculture (200803014) and by the GHI program of Emory University.

\section{Author details}

'State Key Laboratory of Veterinary Biotechnology, Harbin Veterinary Research Institute of Chinese Academy of Agricultural Sciences, Harbin 150001, P.R. China. ${ }^{2}$ Department of Microbiology and Immunology, Emory University School of Medicine, Rollins Research Center, Atlanta, GA 30322, USA.

\section{Authors' contributions}

$\mathrm{LT}, \mathrm{HZ}$ and $\mathrm{TH}$ carried out the plasmid construct cloning, rescued and characterized the recombinant virus. $L T, J G, X W$ and $Z W$ carried out the immunization assay and data analysis. BZ and DK participated in the animal immunization. ZB designed the whole study, provided general supervision and prepared the manuscript. CY participated in experiment design and helped preparing the manuscript. All authors have read and approved the submitted manuscript.

\section{Competing interests}

The authors declare that they have no competing interests.

Received: 16 May 2011 Accepted: 25 September 2011

Published: 25 September 2011

\section{References}

1. Dietzschold B, Schnell M, Koprowski H: Pathogenesis of rabies. Curr Top Microbiol Immunol 2005, 292:45-56.

2. WHO: Rabies. 2009.

3. Song M, Tang $\mathrm{Q}$, Wang DM, Mo ZJ, Guo SH, Li H, Tao XY, Rupprecht CE, Feng ZJ, Liang GD: Epidemiological investigations of human rabies in China. BMC Infect Dis 2009, 9:210

4. Zhang S, Tang $Q$, Wu X, Liu Y, Zhang F, Rupprecht $C E$, Hu R: Rabies in ferret badgers, southeastern China. Emerg Infect Dis 2009, 15(6):946-949.

5. Wilde H, Khawplod P, Khamoltham T, Hemachudha T, Tepsumethanon V, Lumlerdacha B, Mitmoonpitak C, Sitprija V: Rabies control in South and Southeast Asia. Vaccine 2005, 23(17-18):2284-2289.

6. Fu ZF: Rabies and rabies research: past, present and future. Vaccine 1997, 15(Suppl):S20-24.

7. WHO Expert Consultation on rabies First report: WHO Technical Report Series $9312005,1-121$

8. Knobel DL, Cleaveland S, Coleman PG, Fevre EM, Meltzer MI, Miranda ME, Shaw A, Zinsstag J, Meslin FX: Re-evaluating the burden of rabies in Africa and Asia. Bull World Health Organ 2005, 83(5):360-368.

9. Koprowski H, Cox HR: Studies on chick embryo adapted rabies virus; culture characteristics and pathogenicity. J Immunol 1948, 60(4):533-554.

10. Morimoto K, Hooper DC, Carbaugh H, Fu ZF, Koprowski H, Dietzschold B: Rabies virus quasispecies: implications for pathogenesis. Proc Natl Acad Sci USA 1998, 95(6):3152-3156.

11. Ge J, Deng G, Wen Z, Tian G, Wang $Y$, Shi J, Wang $X$, Li Y, Hu S, Jiang $Y$, et al: Newcastle disease virus-based live attenuated vaccine completely protects chickens and mice from lethal challenge of homologous and heterologous H5N1 avian influenza viruses. J Virol 2007, 81(1):150-158.

12. Reed $\amalg$, Muench $\mathrm{H}$ : A simple method of estimating fifty percent endpoints. Am J Hyg 1938, 27.

13. Smith JS: New aspects of rabies with emphasis on epidemiology, diagnosis, and prevention of the disease in the United States. Clin Microbiol Rev 1996, 9(2):166-176.

14. Bellinger DA, Chang J, Bunn TO, Pick JR, Murphy M, Rahija R: Rabies induced in a cat by high-egg-passage Flury strain vaccine. J Am Vet Med Assoc 1983, 183(9):997-998, 965..
15. Fehlner-Gardiner C, Muldoon F, Nadin-Davis S, Wandeler A, Kush J, Jordan LT: Laboratory diagnosis of rabies in Canada for calendar year 2006. Can Vet J 2008, 49(4):359-361.

16. Hosokawa-Muto J, Ito N, Yamada K, Shimizu K, Sugiyama M, Minamoto N: Characterization of recombinant rabies virus carrying double glycoprotein genes. Microbiol Immunol 2006, 50(3):187-196.

17. Faber M, Li J, Kean RB, Hooper DC, Alugupalli KR, Dietzschold B: Effective preexposure and postexposure prophylaxis of rabies with a highly attenuated recombinant rabies virus. Proc Natl Acad Sci USA 2009. 106(27):11300-11305.

18. Faber M, Pulmanausahakul R, Hodawadekar SS, Spitsin S, McGettigan JP, Schnell MJ, Dietzschold B: Overexpression of the rabies virus glycoprotein results in enhancement of apoptosis and antiviral immune response. Journal of virology 2002, 76(7):3374-3381.

19. Tao L, Ge J, Wang X, Zhai H, Hua T, Zhao B, Kong D, Yang C, Chen H, Bu Z: Molecular basis of neurovirulence of flury rabies virus vaccine strains: importance of the polymerase and the glycoprotein R333Q mutation. Journal of virology 2010, 84(17):8926-8936.

20. Mebatsion T, Schnell MJ, Cox JH, Finke S, Conzelmann KK: Highly stable expression of a foreign gene from rabies virus vectors. Proc Natl Acad Sci USA 1996, 93(14):7310-7314.

21. Dietzschold B, Wunner WH, Wiktor TJ, Lopes AD, Lafon M, Smith CL, Koprowski H: Characterization of an antigenic determinant of the glycoprotein that correlates with pathogenicity of rabies virus. Proc Natl Acad Sci USA 1983, 80(1):70-74.

22. Morimoto K, Foley HD, McGettigan JP, Schnell MJ, Dietzschold B: Reinvestigation of the role of the rabies virus glycoprotein in viral pathogenesis using a reverse genetics approach. J Neurovirol 2000, 6(5):373-381

23. Morimoto K, Hooper DC, Spitsin S, Koprowski H, Dietzschold B: Pathogenicity of different rabies virus variants inversely correlates with apoptosis and rabies virus glycoprotein expression in infected primary neuron cultures. Journal of virology 1999, 73(1):510-518.

doi:10.1186/1743-422X-8-454

Cite this article as: Tao et al: Generation of a recombinant rabies Flury LEP virus carrying an additional $G$ gene creates an improved seed virus for inactivated vaccine production. Virology Journal 2011 8:454.

\section{Submit your next manuscript to BioMed Central and take full advantage of:}

- Convenient online submission

- Thorough peer review

- No space constraints or color figure charges

- Immediate publication on acceptance

- Inclusion in PubMed, CAS, Scopus and Google Scholar

- Research which is freely available for redistribution 